\title{
Confluence and cold shut computation based on time field in casting simulation
}

\author{
Yong-shuai Feng', *Dun-ming Liao', Tao Chen ${ }^{2}$ \\ 1. State Key Laboratory of Materials Processing and Die \& Mould Technology, School of Materials Science and Engineering, Huazhong \\ University of Science and Technology, Wuhan 430074, China; \\ 2. Wuhan Chenxi Yunfeng Technology Co., Ltd., Wuhan 430074, China
}

\begin{abstract}
Numerical simulation technology has been widely used in the foundry industry to analyze and improve casting processes. During the casting filling process, many filling-related defects form easily at the confluences of liquid metal streams. The main filling-related defects are cold shut defects. To calculate the positions of casting defects, the characteristics of liquid metal confluences were analyzed. The flow front of liquid metal was captured by the volume-of-fluid algorithm to obtain a time field, which was used to calculate the time derivatives of the liquid front position and the confluences of liquid metal streams. To distinguish small confluences from the main confluences, the concept of confluent scale was developed, which was used to filter the small confluences based on a threshold. The calculation process was demonstrated through the post-processing of numerical simulation. A "W" shaped casting and a steering wheel casting were calculated to validate the accuracy of the method developed in this study. The positions of cold shut defects were predicted by calculating the confluences of liquid metal streams. The method was proved to be practical by comparing the calculation results with the positions of cold shut defects in an end cover casting. The computation of confluences and cold shut defects can improve the analysis efficiency and provide assurance for the optimization of a casting process plan.
\end{abstract}

Key words: numerical simulation; casting defects; flow front; time field; confluence; cold shut

CLC numbers: TP391.9 Document code: A

Article ID: 1672-6421 2021)02-101-09

\section{Introduction}

Numerical simulations have been widely used in practical casting process development. The filling process, solidification process, defect formation, and distribution characteristics during the casting process can be predicted by numerical simulation before actual experiments ${ }^{[1,2]}$. Generally, numerical simulations have three main components: pre-processor, solver, and post-processor. Process designers analyze and judge whether the casting process is reasonable by examining the results after post-processing ${ }^{[3,4]}$. The process of observing and analyzing casting defects is complicated and difficult, not only creating a huge workload but also facing problems of occlusion and visualization difficulties in three-dimensional space ${ }^{[5]}$. Researchers have investigated the casting defects using numerical

\section{*Dun-ming Liao}

Male, born in 1973, Ph.D, Professor. His research interest mainly focuses on casting process simulation, especially on casting stress simulation and casting process CAD

E-mail: liaodunming@hust.edu.cn;

Received: 2020-11-16; Accepted: 2021-02-22 simulation. Niyama et al. ${ }^{[6]}$ proposed the Niyama criterion in 1982 to calculate shrinkage defects during the solidification process. The criterion can accurately predict shrinkage and has been widely used in numerical simulations ${ }^{[7,8]}$. Jia et al. ${ }^{[9]}$ predicted the shrinkage during a casting process by dynamically dividing the isolated domain. Kang et al. ${ }^{[10]}$ used multiple thermal nodes and shrink-and-compensate methods to calculate and display the positions of shrinkage defects. Zhou et al. ${ }^{[11]}$ used a time gradient method to calculate the feed path and local hot points during solidification. Sutaria et al. ${ }^{[12]}$ used the level-set-method to calculate the solidification filling path and predicted shrinkage.

To date, the studies of simulating casting defects have tended to focus on solidification and cooling processes rather than filling process. There has not been much research on the numerical simulation of filling-related defects. However, some significant casting defects occur during the filling process. The confluences of liquid metal streams are the key positions where casting defects frequently occur during the filling process. Casting defects, such as those caused by flow turbulence and trapped gases, occur when the flow velocity of liquid metal is too fast at the confluence. During the 
filling process of lost foam casting, the pyrolysis products are trapped at the confluence regions, and eventually irregular stripes or spots are formed on the casting surface ${ }^{[13]}$. Cold shut defects may occur if the temperature at the confluence is too low ${ }^{[14-16]}$, and they often occur on the surfaces and thin walls of a casting far from the runner ${ }^{[17-18]}$. The main reason for their occurrence is that two liquid metal streams become semi-solid when they merge, and consequently, they cannot completely merge ${ }^{[19-21]}$. Cold shut defects reduce the surface accuracy and mechanical properties of the cast component ${ }^{[22-25]}$. The positions of cold shut defects can be predicted by calculating the confluence of liquid metal.

In this study, the characteristics of liquid metal flow during the casting filling process were analyzed and the flow front of liquid metal was calculated based on the volume-of-fluid (VOF) algorithm to obtain a time field. Then, the confluence position of liquid metal was calculated based on the time field. To distinguish small confluences from the main confluence, the computation method has been modified and the concept of a confluent scale was developed. Small-scale confluences can be filtered by setting a threshold so that only the main confluence is found in the post-processing. A "W" shaped casting was used to explain the calculation method of the main confluence. A steering wheel casting was used to verify the accuracy of the proposed algorithm. The positions of the cold shut defects were predicted by calculating the main confluences of an end cover casting. The calculation results are consistent with the experimental result, which verified the accuracy of the adopted model.

\section{Analysis and computation of confluence}

\subsection{Analysis of confluence}

During the filling process of a casting, it is difficult to locate the confluences of liquid metal streams. The confluences can only be observed before the collision of liquid metal by numerical simulations. However, the confluent positions are challenging to be found because the flow state of liquid metal at a confluence changes after the collision, and the time and position of the collision are uncertain. By observing and analyzing the flow of liquid metal, it can be easily determined that a confluence of liquid metal is the position where the flow front of two liquid streams converge. The confluence is only related to the flow front of liquid metal. Therefore, it is necessary to follow the flow front positions of the liquid metal to find the confluences. The algorithms of front-tracking and front-capturing for fluid are fully mature, and the most widely used interface front extraction algorithm is the volume-of-fluid (VOF) algorithm ${ }^{[26,27]}$. A volume function $F$ is defined in the VOF algorithm as the ratio of the liquid metal volume in the grid $\left(V_{l}\right)$ to the grid volume $(V)$ :

$$
F(x, y, z, t)=\frac{V_{l}(x, y, z, t)}{V(x, y, z, t)}
$$

where $F$ is the volume fraction of the liquid metal. The value of $F$ is 1 when the grid is full of liquid metal, and $F$ equals to zero when the grid is full of air. When the value of $F$ is between 0 and 1 , it means that the grid is the interface front of liquid metal. The continuity equation is as follows:

$$
\frac{\partial \rho}{\partial t}+\frac{\partial(\rho u)}{\partial x}+\frac{\partial(\rho v)}{\partial y}+\frac{\partial(\rho w)}{\partial z}=0
$$

The volume-fraction equation governs the distribution rule of $F$ :

$$
\frac{\partial F}{\partial t}+\nabla \cdot(F \vec{u})=0
$$

The velocity can be calculated by Navier-Stokes equations:

$$
\rho \frac{\partial \vec{u}}{\partial t}+\rho \vec{u} \cdot \nabla \vec{u}=-\nabla p+\mu \nabla^{2} \vec{u}+\vec{f}
$$

where $\rho$ is the liquid density, $t$ is the time, $u, v$ and $w$ are the velocity in the $x, y$ and $z$ direction, respectively, $p$ is the pressure, $\vec{u}$ is velocity of the vector, $\vec{f}$ is the body force due to gravity, and $\mu$ denotes dynamic viscosity.

To facilitate the analysis and explanation of the problem, the confluence in the two-dimensional case was analyzed. Two liquid metal streams were assumed to flow along the $x$-axis. The position of the liquid-air interface was calculated at different times $t$. The time $t$ when the liquid metal front passes through a grid cell was recorded. This value increases along the direction of streamlines. The results before a confluence are shown in Fig. 1(a), and the results after a confluence are shown in Fig. 1(b). The two-dimensional surface of time field is shown in Fig. 1(c), in a saddle shape. As shown in Fig. 1(c), the time value increases firstly and then decreases along the $x$ direction, and the maximum value appears near the $y$-axis. The position of the $y$-axis is the confluence of liquid metal. The $x 0 t$ slice of Fig. 1(c) is shown in Fig. 1(d), which shows the change of the time value along the $x$-axis, and the position of the peak is the confluence. According to the above analysis of the confluence, it is necessary to calculate the positions of the flow front at different times to obtain a time field, and then calculate the position of the local peak according to the time derivative value to obtain the confluence of liquid metal.

\subsection{Computation of confluence}

Many influential factors are involved in the computation of the confluence, and the following assumptions are proposed to simplify the problem:

(1) The time step is sufficiently small $(\Delta t \rightarrow 0)$. More specifically, to ensure temporal continuity, the distance at which the liquid metal flows forward in each time step is not greater than one grid's length.

(2) The flow front of liquid metal is stable, and there is no splashing during the flow process.

(3) The liquid-air interface is a convex curved surface, and there is no recessed position.

To calculate the confluence, the time function should be obtained based on the position of the flow front of the liquid metal. According to the volume function $F$ at different times $t$, the coordinates $(x, y, z)$ of the grid position of the flow front 
(a)

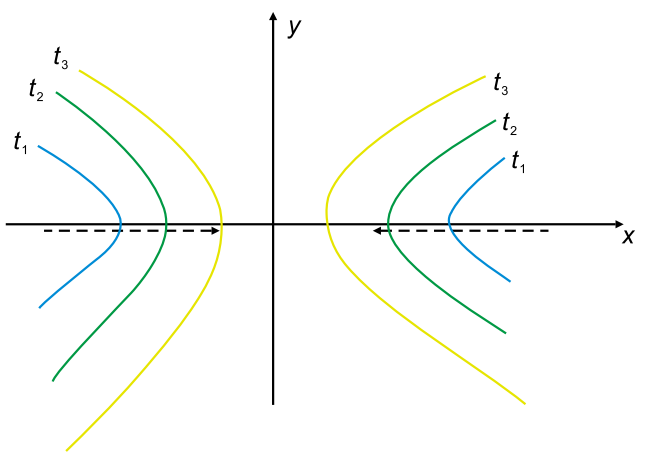

(c)

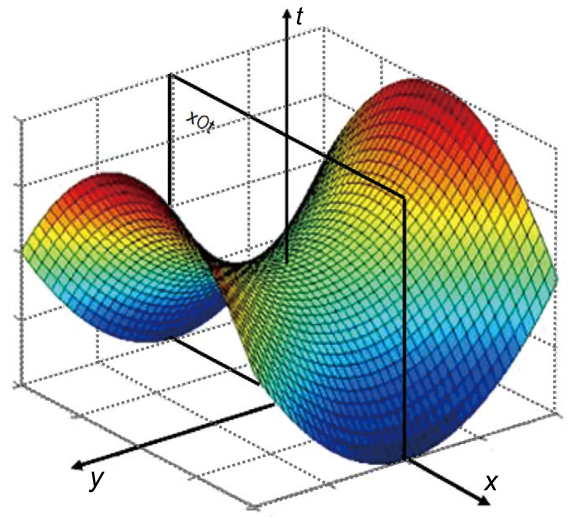

(b)

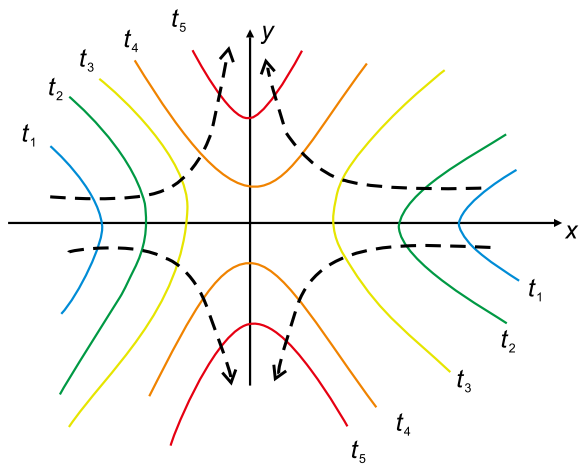

(d)

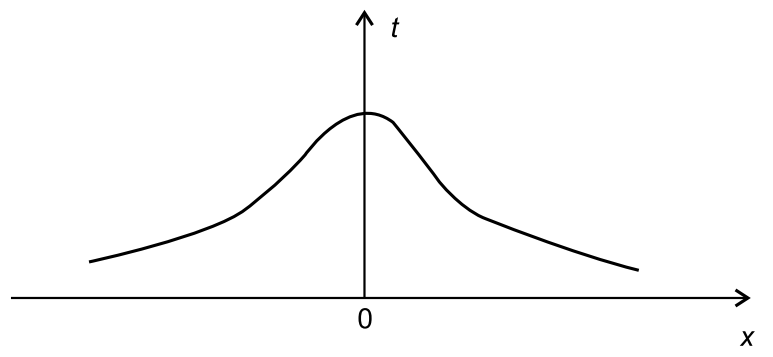

Fig. 1: Analysis of confluence in two-dimensions: (a) result before confluence; (b) result after confluence; (c) time field result; (d) time value along $x$-axis in $x 0 t$ slice in (c)

can be obtained, and the time function $T$ can be obtained as follows:

$$
T(x, y, z)=t \quad(F(x, y, z, t-\Delta t)=0, F(x, y, z, t)>0)
$$

The time value $t$ at which the liquid-air interface arrives at a grid cell is stored. In the one-dimensional case, the calculation process is shown in Fig. 2. Assuming that a stream of liquid metal flows along the $x$-axis, the first flow field shows the liquid metal at time $t_{1}$, then the value of the flow front grid $x_{1}$ is set to be $t_{1}$, and the value of $T\left(x_{1}\right)$ equals to $t_{1}$. The second flow field shows the flow at time $t_{2}$, and the value $T\left(x_{2}\right)$ is set to be $t_{2}$. By continuing this process, the time field can be obtained.

An aluminum casting was used to demonstrate the calculation of the time field. Figure 3 shows the filling process of the liquid metal. Figure 4 is the calculation result of the time field by the extraction of liquid-air interface. The attribute value is time value, and the greater the value, the later the liquid metal front passes through this location. By extracting the position of the flow front, the time-varying

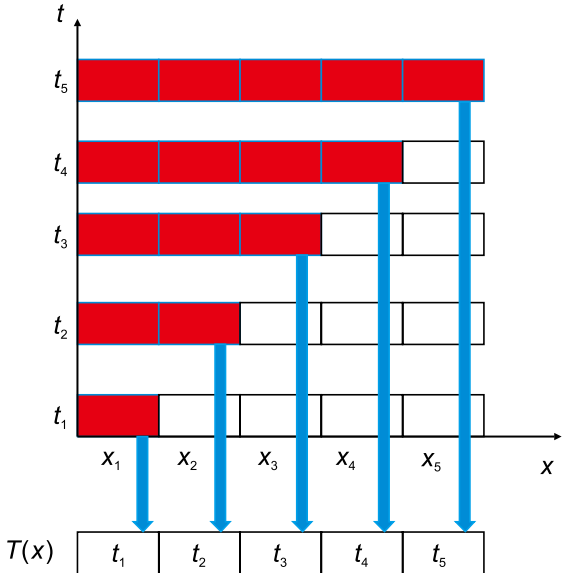

Fig. 2: Calculation of time field

flow field can be compressed into a fixed and constant time field. This compresses the data and dramatically reduces the required amount of data. Integer values of the time were used to replace the specific time value $t$ represented by the floating-
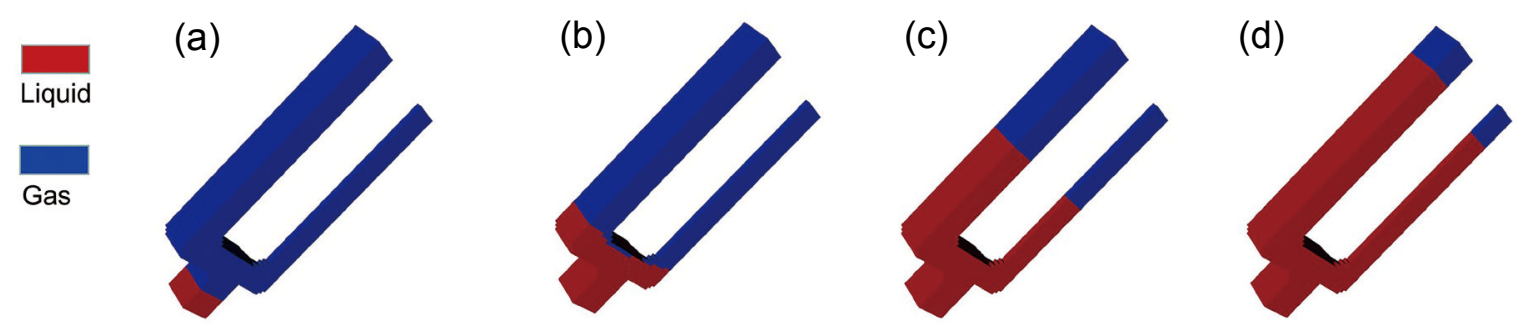

Fig. 3: Filling process of an aluminum casting 

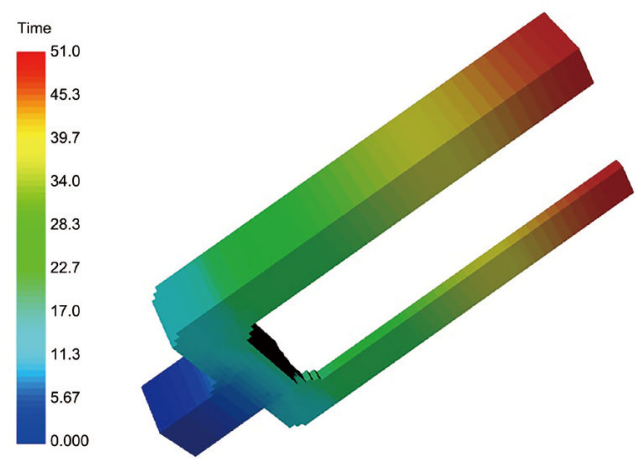

Fig. 4: Calculated time field for aluminum casting by extraction of liquid-air interface

point number for storage. This significantly decreases the memory consumption and calculation time, leading to a higher calculation efficiency.

The position of the confluence can be roughly determined after the calculation of the time field. Then, the time derivative needs to be calculated to obtain the confluent positions of the liquid metal. According to the analysis in Section 2.1, it can be concluded that the condition for the confluence of liquid metal is that the time function has a maximum value in one dimension, and the position of the maximum value of the time field is the confluence of liquid metal. The criterion to determine the position of the maximum value is as follows:

$$
T^{\prime \prime}(x)<0
$$

The different forms of the first and second derivatives are

$$
\left\{\begin{array}{l}
T^{\prime}(x)=\lim _{\Delta x \rightarrow 0} \frac{T(x+\Delta x)-T(x)}{\Delta x} \\
T^{\prime \prime}(x)=\lim _{\Delta x \rightarrow 0} \frac{T^{\prime}(x+\Delta x)-T^{\prime}(x)}{\Delta x}
\end{array}\right.
$$

and thus, the Eq. (6) changes to

$$
\lim _{\Delta x \rightarrow 0} \frac{T(x-\Delta x)+T(x+\Delta x)-2 T(x)}{\Delta x^{2}}<0
$$

A one-dimensional case is used for analysis (Fig. 5). It is assumed that two liquid metal streams flow in opposite directions along the $x$-axis. Eventually, the liquid streams converge at the intermediate position $a$. The time value $T(x)$ stored in the grid is a function of the coordinate $x$. The grid cell on the left of the confluence was selected for analysis. Assuming that the coordinates of this position are represented by $a_{1}$, the grid cell corresponding to $a_{1}$ does not satisfy the condition of the confluence [Eq. (8)]. The grid cell $a_{2}$ that is on the right of the confluence also does not meet the condition of the confluence. At the confluence $a$, the grid $a$ meets the condition of the confluence, and the confluence can be obtained.

The three-dimensional case is similar to the onedimensional case above. In the three-dimensional space, the

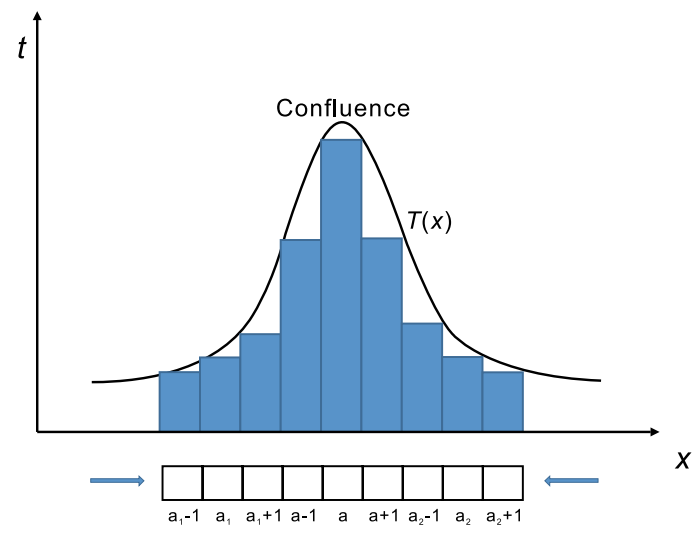

Fig. 5: Process of calculating confluence in one dimension

newly appearing liquid metal flow front surrounds the flow front that appears at the previous moment. When the confluence is detected, if check in the reverse direction of the flow, the value of the time field shows a continuous decreasing trend. The position of confluence still has the maximum value of time field, which still satisfies the condition of calculation. A die casting process is shown in Fig. 6. Figure 6(a) illustrates the geometric model of the casting with two inlets. The liquid metal flows from the two inlets into the mold and converges in the middle of the mold [Fig. 6(b)]. The calculation result of the time field is shown in Fig. 6(c). The position that connects the two streams of liquid metal together is the confluence. Any point in the confluent surface still meets the one-dimensional confluence feature extraction conditions mentioned above. The three-dimensional case is expanded based on one-dimensional case. The detection direction is expanded from one direction to three directions. The second-order partial derivatives of the time function in the $x, y$, and $z$ directions in three-dimensional space are less than zero [Eq. (9)], the difference form is Eq. (10). If one of the three conditions is satisfied, the position is the confluence.

$$
\left\{\begin{array}{l}
\frac{\partial^{2} T}{\partial x^{2}}<0 \\
\frac{\partial^{2} T}{\partial y^{2}}<0 \\
\frac{\partial^{2} T}{\partial z^{2}}<0
\end{array}\right.
$$

$$
\left\{\begin{array}{l}
\frac{\partial^{2} T}{\partial x^{2}}=\lim _{\Delta x \rightarrow 0} \frac{T(x-\Delta x, y, z)+T(x+\Delta x, y, z)-2 T(x, y, z)}{\Delta x^{2}} \\
\frac{\partial^{2} T}{\partial y^{2}}=\lim _{\Delta y \rightarrow 0} \frac{T(x, y-\Delta y, z)+T(x, y+\Delta y, z)-2 T(x, y, z)}{\Delta y^{2}} \\
\frac{\partial^{2} T}{\partial z^{2}}=\lim _{\Delta z \rightarrow 0} \frac{T(x, y, z-\Delta z)+T(x, y, z+\Delta z)-2 T(x, y, z)}{\Delta z^{2}}
\end{array}\right.
$$

\subsection{Modifying the computation of confluence}

Ideally, the time step $(\Delta t)$ is small enough and the liquid metal does not flow more than the distance of one grid in one time step [Fig. 7(a)]. 
(a)

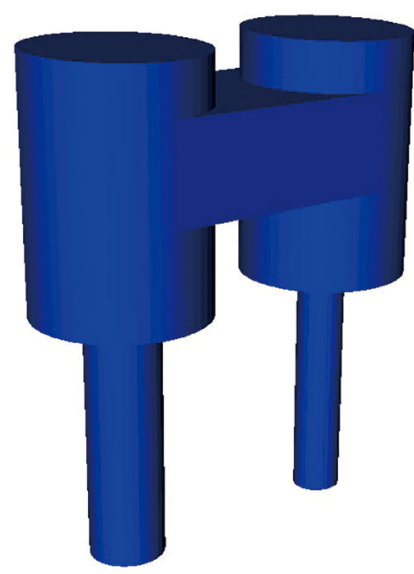

(b)

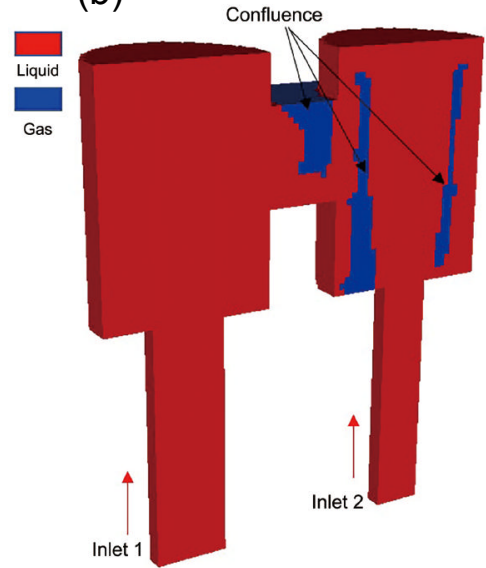

(c)

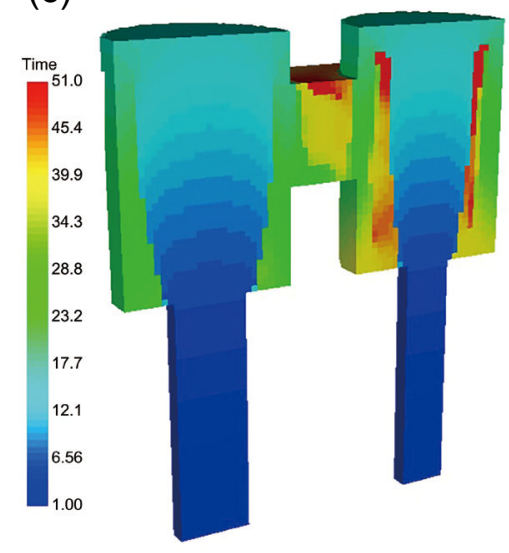

Fig. 6: Confluence in three dimensions:

(a) casting mold; (b) filling process; (c) time field

However, the time step cannot be small enough in practice, which cannot satisfy ideal assumptions. Consequently, the fluid front of liquid metal moves by more than one grid cell in a time step. If the time step is not small enough, the area of the interface that flows during one time step is regarded as the flow front area, as shown in Fig. 7(b). This means the time values of multiple consecutive adjacent grids are the same along the flow direction. If the computation is still performed according to the above extraction conditions, some confluent positions cannot satisfy the extraction conditions and are ignored. The confluence can be detected in Fig. 7(a) but cannot be detected in Fig. 7(b). (a)

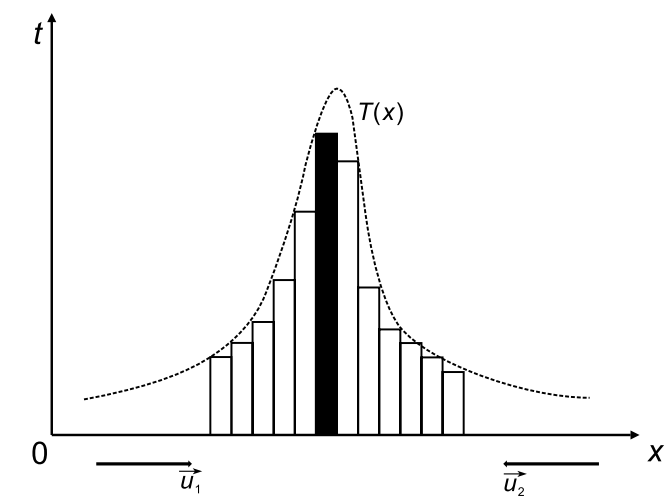

(b)

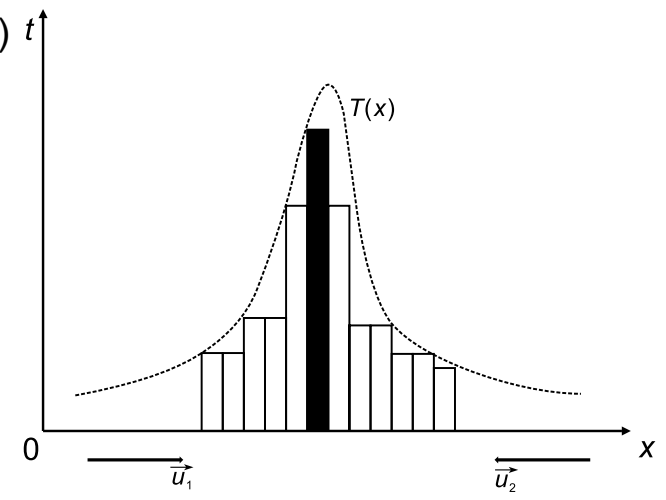

Fig. 7: Influence of time step on confluence

To solve this problem, the extraction conditions for the confluence should be relaxed appropriately. When the secondorder difference result of the grid is less than zero [Eq. (11)], the position meets the condition and is the confluence. If the second-order difference result of the grid is greater than zero [Eq. (12)], the position is not the confluence. When the second-order difference of the grid is equal to zero [Eq. (13)], no conclusion can be drawn. It is necessary to verify the position again after $\Delta x$ is gradually increased. In this study, if the result of the second-order difference is zero, $\Delta x$ increases the length of one grid cell, and can increase up to five grid cells. If $\Delta x$ increases to the length of five grid cells and the second-order difference is still equal to zero, the position is not considered to be the confluence. As shown in Fig. 8, if the size of $\Delta x$ is the length of one grid, the range of the second-order difference is $d x_{1}$. The result of the second-order difference is zero, and the condition of the confluence cannot be met, so the confluence cannot be detected. When the value of $\Delta x$ increases to the length of two grid cells, the range of the second-order difference is $d x_{2}$. The condition of the confluence can then be met, and the confluence of liquid metal can be detected. Therefore, to solve the problem of a large time step, the extraction conditions of the confluence can be appropriately relaxed to realize the extraction of the confluence.

$$
\begin{aligned}
\frac{T(x-\Delta x)+T(x+\Delta x)-2 T(x)}{\Delta x^{2}}<0 & \\
\frac{T(x-\Delta x)+T(x+\Delta x)-2 T(x)}{\Delta x^{2}}>0 & \\
\frac{T(x-\Delta x)+T(x+\Delta x)-2 T(x)}{\Delta x^{2}} & =0
\end{aligned}
$$

The interface front of liquid metal is assumed a convex curved surface in Section 2.2. However, during the filling process of the casting, the assumption is hard to satisfy. Therefore, it is necessary to further improve the computation method in Section 2.2. During the filling process of the casting, 


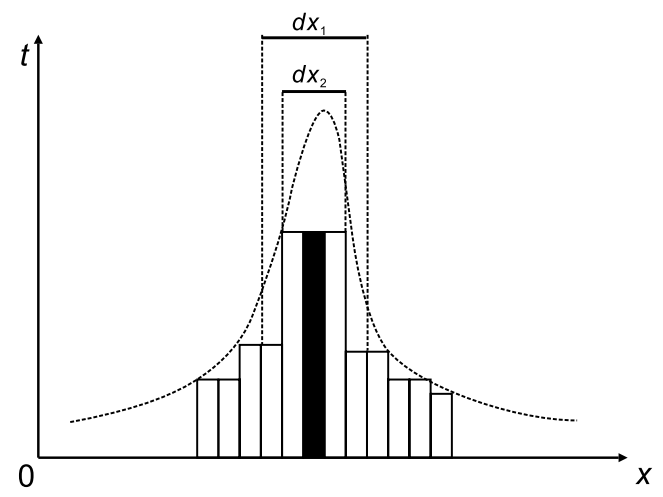

Fig. 8: Influence of detection length

the leading edge of liquid metal is not an ideal convex surface. At the position of the leading-edge, the liquid metal fluctuates greatly and forms a complex irregular three-dimensional curved surface. As shown in Fig. 9(a), due to the fluctuation at the leading edge of the liquid metal, a liquid metal stream forms multiple small liquid metal streams at different positions of the flow front. These small liquid streams also converge. This kind of confluence always appears during the process of filling. These small confluences also can meet the conditions for extracting the confluence mentioned above. These small confluences of the fluid flow may be extracted, but it is evident that they are not the positions being searched for. To distinguish the small confluences from the main confluences, the concept of "confluent scale" was proposed as a reference quality. Using the time function $T$, the continuous decrease of the time value with the distance from the position of the confluence along the reverse direction of the liquid metal flow was calculated and the smaller value was selected as the value of confluent scale $L$. Starting from the position of the confluence, the detection distance increases in the reverse direction of the flow. If the time value increases, the value of the confluent scale $L$ is the detection distance at this time. As shown in Fig. 9(b), the descending distance from the Confluence a is the distance of three grid cells, and thus, the value of the confluence scale $L$ is selected as 3. Similarly, the descending distance from the Confluence $b$ is the distance of five grid cells, then the value of confluence scale is 5 , and the same, the confluence scale of Confluence $\mathrm{c}$ is 2 . To remove the small confluences, a threshold was used to filter the results. The confluences whose scales are smaller than the threshold can be removed, only the large-scale confluences are left. If the threshold value is greater than 2 , the confluence caused by the fluctuations can be filtered out. For different castings, the threshold should be generally set at $1 \%$ of the maximum casting length, which can filter out the small confluences. The general calculation process is shown in Fig. 10.

\section{Results analysis and cold shut prediction}

\subsection{Analysis and verification of calculation result}

To explain the results conveniently, a casting with a "W" shape was analyzed. The results of the velocity field (Fig. 11) show that the liquid metal flows into the mold from both sides and converges at the middle position of the mold. The liquid metal fills the mold upward after the collision [Fig. 11(e)]. Figure 12(a) shows the time field values when the liquid metal front area reaches the grid cells. Figure 12(b) shows the result of the confluence on the surface of the casting, and the areas in red indicate the confluence. The liquid metal continues to form small confluences at the flow front area during the filling (a)

\section{Confluence}

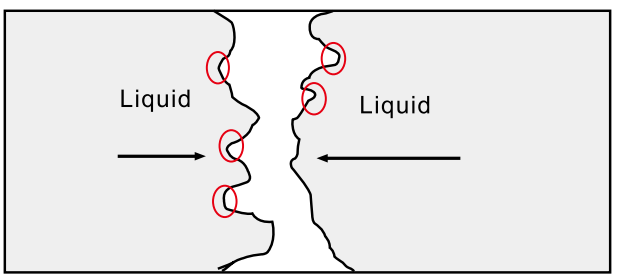

(b)

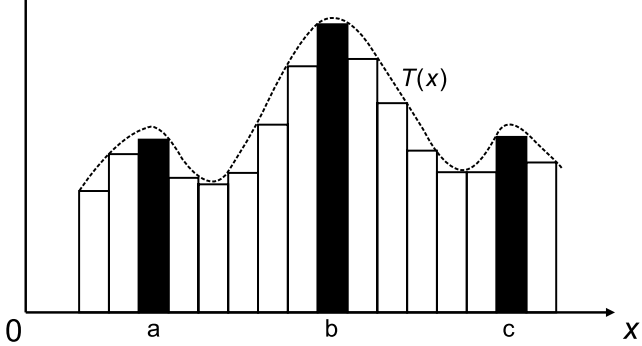

Fig. 9: Unstable flow of leading edge

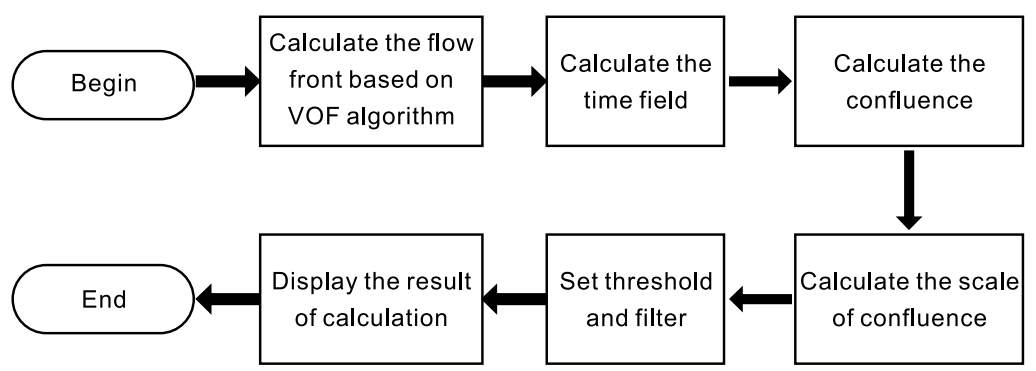

Fig. 10: Algorithm for extracting confluence 


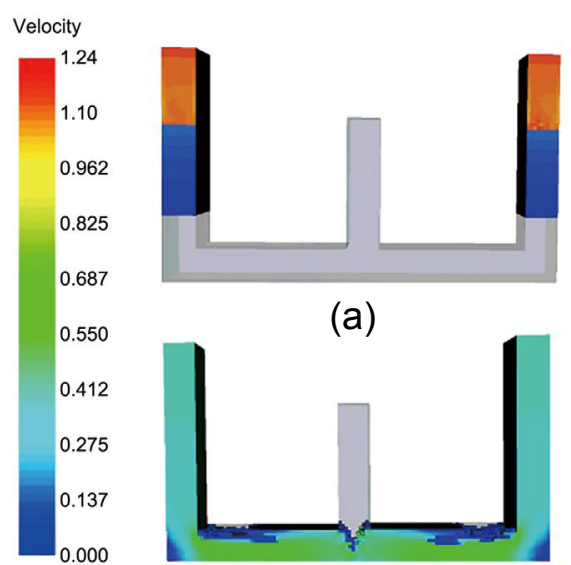

(d)

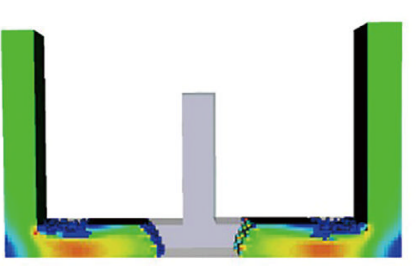

(b)

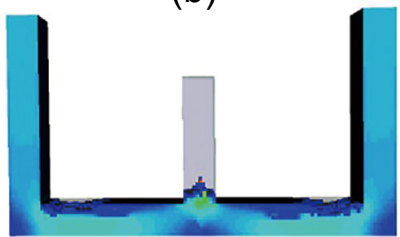

(e)

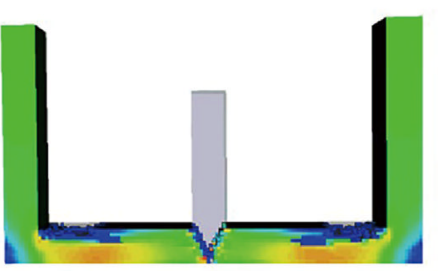

(c)

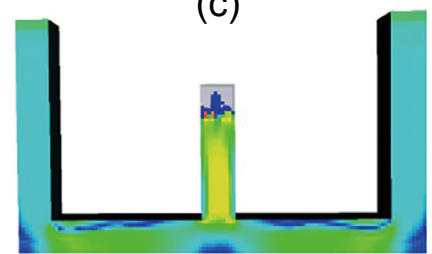

(f)

Fig. 11: Velocity field of filling process

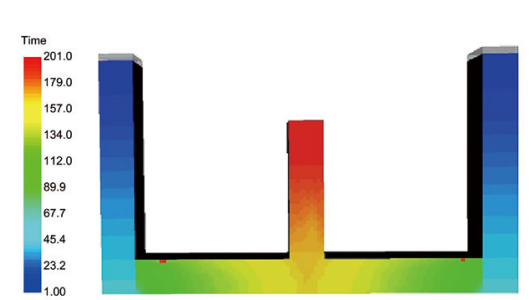

(a)

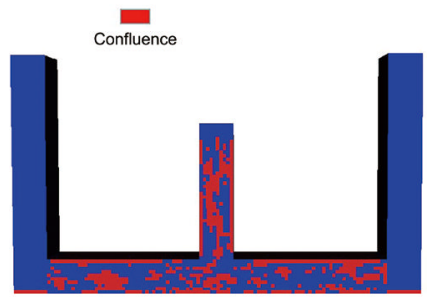

(b)

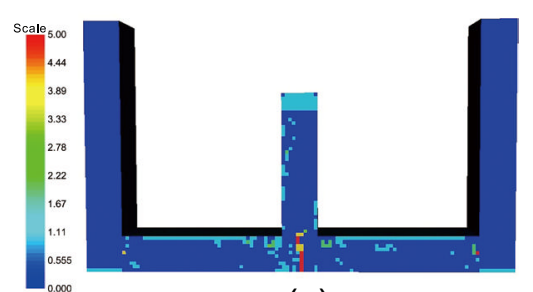

(c)

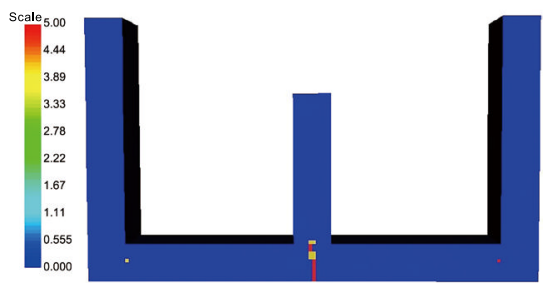

(d)

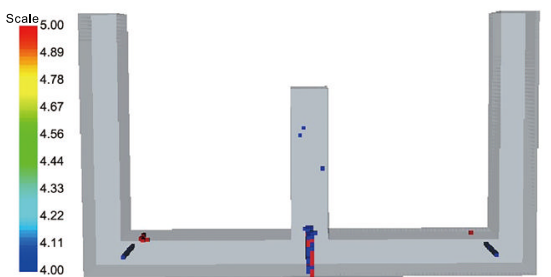

(e)

Fig. 12: Analysis results of time field of casting (a), confluence of casting (b), confluent scale (c), main confluence of casting surface (d), and main confluence results (e)

process. Figure 12(c) illustrates the confluent scale. It can be seen that the value at the middle of the mold is significantly greater than the others. Figure 12(d) shows the result of the main confluence on the surface of the casting. The small-scale confluences are removed by setting a threshold. Comparing the confluence results with the velocity field results during the filling process, the confluence can be clearly observed. After the liquid converges, the liquid metal reflows to both sides. The reflowed liquid metal converges again on both sides. Figure 12(e) shows the result of removing the non-confluence area and the smaller confluences. By comparing the velocity field result of the filling process (Fig. 11) with the calculation results [Fig. 12(d) and Fig. 12(e)], the rationality and accuracy of the proposed algorithm could be verified.

\subsection{Calculation example}

Figure 13 shows the simulation results of the casting process of a steering wheel casting. The liquid metal flows from the pouring gate at high pressure and flows through the gating system into the casting mold. Figure 13 shows the velocity of the liquid metal in the mold at different times during the filling process. As shown in Fig. 13(a), there is a large confluent scale. The liquid metal that flows from different routes of the gating systems converges at the center of the mold. The liquid metal also converges at the junctions of the mold [Fig. 13(b)]. Figure 13(c) shows two main confluent positions, and in Fig. 13(d), the liquid metal streams finally converge in the mold. Figure 14 shows the extracted confluent positions formed at different times during the liquid metal filling process. The primary six confluent positions were accurately calculated by the proposed algorithm and the attribute value was the confluence scale.

\subsection{Cold shut prediction}

Figure 15 shows a casting of an end cover. The numerical simulation result of the filling process is shown in Fig. 15(a). Two end cover castings were made under the same conditions. The liquid metal flows into the casting mold from eight different channels and converges in the mold. Some cold shut defects appear on the surface of the casting [Fig. 15(b)]. The cold shut defects form because the temperature of the confluence is too 
(a)

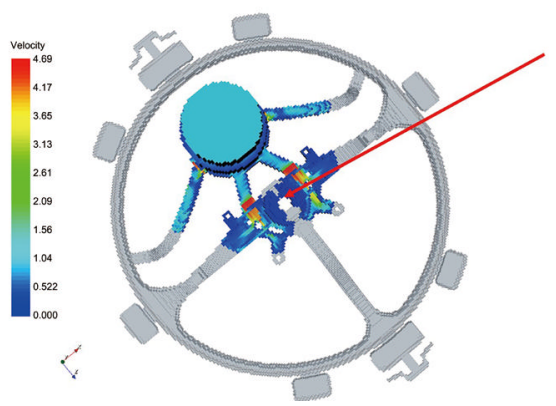

(c)

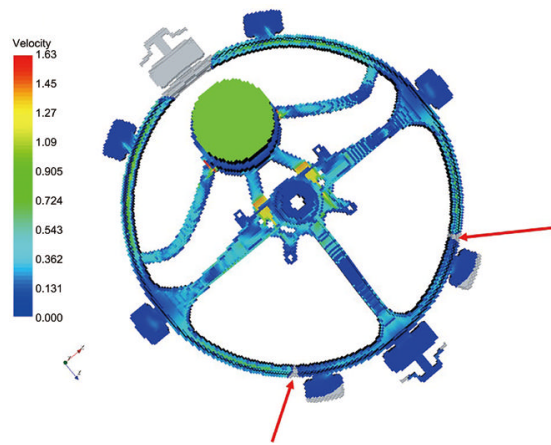

(b)

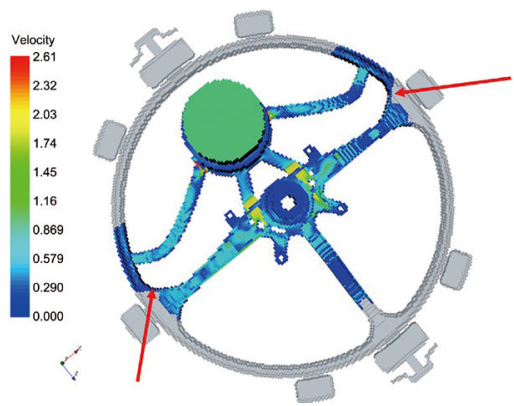

(d)

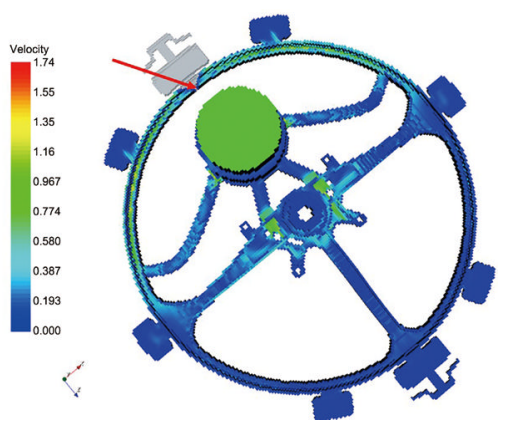

Fig. 13: Filling process of a steering wheel casting
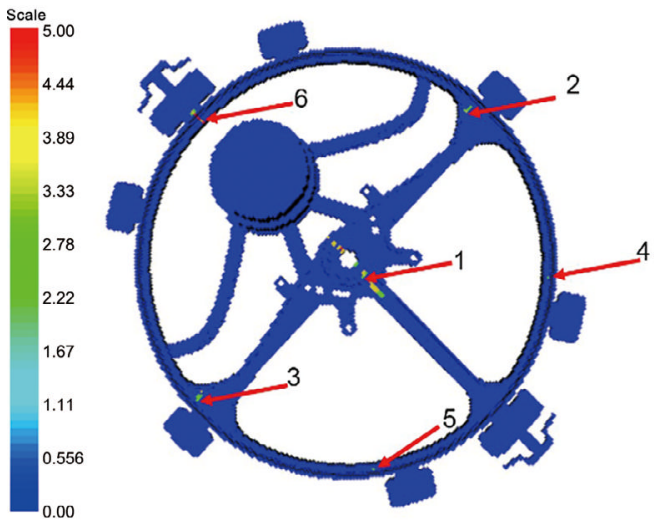

(1)

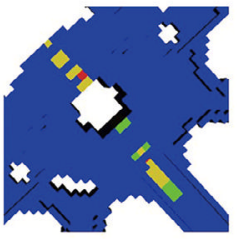

(4)

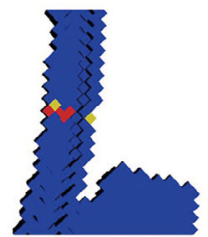

(2)

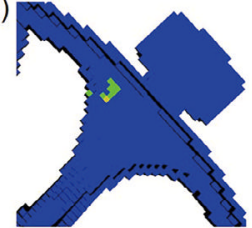

(5)

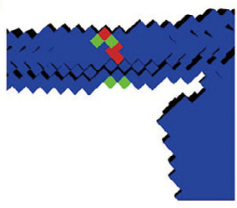

(3)

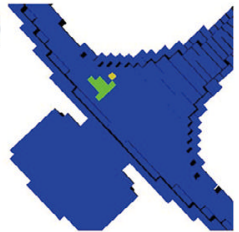

(6)

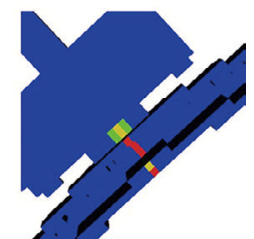

Fig. 14: Result of main confluence on surface of casting

(a)

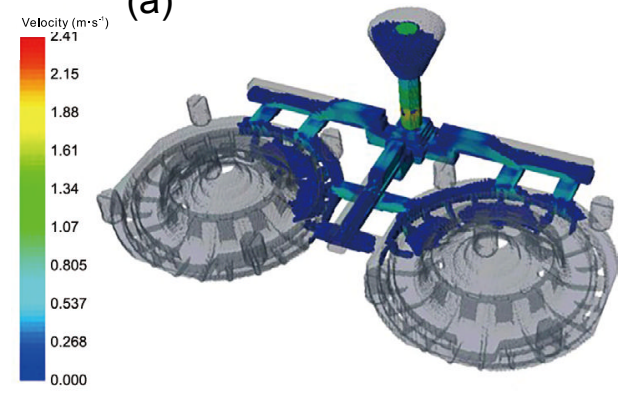

(b)

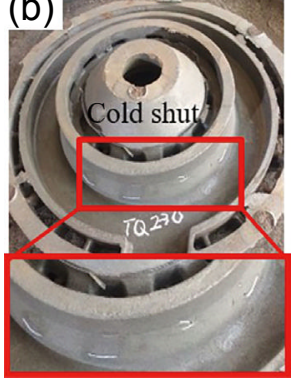

(c)

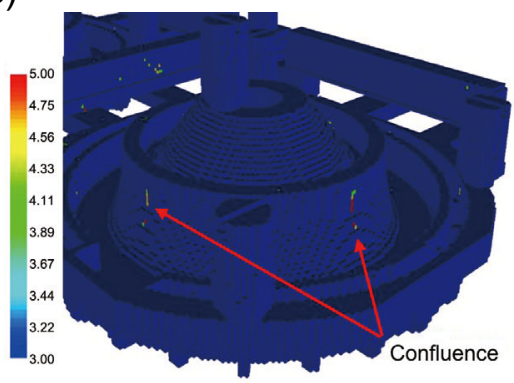

Fig. 15: End cover casting: (a) filling process; (b) cold shut defects; (c) confluences of liquid metal

low and the liquid metal could not completely fuse together. Figure 15(c) shows the main confluences calculated by the proposed algorithm. The cold shut defects form easily at the confluences that far from the flow path. The calculated result is consistent with the experimental results, which verifies the accuracy of the proposed algorithm. 


\section{Conclusions}

(1) During the filling process of casting, the confluences of liquid metal are often prone to defects such as gas entrainment, slag inclusion, and cold shut. This study analyzed the time field and proposed the solution conditions of the confluences. The computation of confluences is helpful for process analysis and prevents engineers from placing the confluence at essential parts, preventing filling-related defects from forming at important parts of the castings.

(2) Due to the unstable flow of liquid metal in the filling process, the flow front area may continue to form small confluences. To distinguish these small confluences from the large confluences in the mold, the concept and calculation method of the confluent scale are proposed, by which the confluence more easily distinguished. The small local confluence formed due to the unstable flow of the liquid metal is filtered by setting a threshold, then, only the main confluence is highlighted and retained.

(3) Using the proposed computation method of the confluences, the cold shut defects in an end cover casting were successfully predicted, and the calculation result was consistent with the experimental results, which further verifies the accuracy of the proposed algorithm. Therefore, the computation method in this study can accurately predict the location of cold shut defects, and provide basis and assurance for the casting process optimization.

\section{Acknowledgements}

This study was supported by the National Key Research and Development Program of China (Nos. 2020YFB2008300 and 2020YFB2008302) and the Program of Key Research and Development Program of Guangdong Province (No. 2019B090921001).

\section{References}

[1] Ravi B, Sivaraman S, Sasikumar R, et al. Indigenous development and industrial application of metal casting simulation software. Transactions of the Indian Institute of Metals, 2015, 68(6): 1227-1233.

[2] Chen T, Liao D M, Shen X, et al. Application of implicit surface in post-processing of casting simulation. The International Journal of Advanced Manufacturing Technology, 2013, 69(5-8): 1841-1854.

[3] Bruna M, Bolibruchová D, Pastirčák R, et al. Gating system design optimization for investment casting process. Journal of Materials Engineering and Performance, 2019, 28(7): 1-7.

[4] Huang P H, Lin C J. Computer-aided modeling and experimental verification of optimal gating system design for investment casting of precision rotor. The International Journal of Advanced Manufacturing Technology, 2015, 79(5-8): 997-1006.

[5] Wang H H. Research on key techniques of feature-based texture visualization for complex flow field. Ph.D. Thesis, Hunan: Graduate School of National University of Defense Technology, 2015: 3-6. (In Chinese)

[6] Niyama E, Uchida T, Morikawa M, et al. A method of shrinkage prediction and its application to steel casting practice. Imono, 1982, 54(8): 507-517.
[7] Monroe C, Beckermann C. Prediction of hot tearing using a dimensionless Niyama criterion. JOM, 2014, 66(8): 1439-1445.

[8] Carlson K D, Beckermann C. Prediction of shrinkage pore volume fraction using a dimensionless Niyama criterion. Metallurgical and Materials Transactions A, 2009, 40(1): 163-175.

[9] Jia B Q, Xiong S M, Liu B C. Dynamical division of isolated melting zone and numerical simulation of shrinkage pore and porosity during solidification of casting. Foundry Technology, 1996, 17(5): 15-17. (In Chinese)

[10] Kang J W, Xiong S M, Liu B C. Study on the prediction of shrinkage cavity in steel casting by multi-hot-spot and simultanous shrinkage feeding method. Foundry, 2000, 49(8): 478-481. (In Chinese)

[11] Zhou J X, Wang M, Yin Y J, et al. Feed paths and hot spots computation based on a time gradient method in casting. The International Journal of Advanced Manufacturing Technology, 2017, 93(1-4): 261-272.

[12] Sutaria M, Gada V H, Sharma A, et al. Computation of feedpaths for casting solidification using level-set-method. Journal of Materials Process Technology, 2012, 212(6): 1236-1249.

[13] Wan X Z. Effect of pouring temperature on the defects of aluminum alloy lost foam castings. Foundry, 2013, 62(3): 206209. (In Chinese)

[14] Kumar J Y, Amirthagadeswaran K S, Gowrishankar S. Casting process optimization for reducing the cold shut defect in castings using response surface methodology. Indian Journal of Engineering \& Materials Sciences, 2015, 22(2): 187-194.

[15] Rajkolhe R, Khan J G. Defects, causes and their remedies in casting process: a review. International Journal of Research in Advent Technology, 2014, 2(3): 375-383.

[16] Jiang D P, Yao Z X. Analysis and measure of eliminating cold shut in Mg-alloy die castings. Foundry Technology, 2016, 37(11) 2518-2520. (In Chinese)

[17] Zhao X Q, Wang G J, Sun Z R, et al. Casting defects often occurring with gray iron engine cylinder block and their prevention measures. Gray Iron, 2014, 34(1): 44-50. (In Chinese)

[18] Zhu H B, Zhang H, Wang L P, et al. The processing improvement study of LPT blade with slender body. Special Casting \& Nonferrous Alloys , 2019, 39(8): 23. (In Chinese)

[19] Cao L, Liao D M, Sun F, et al. Numerical simulation of cold-lap defects during casting filling process. The International Journal of Advanced Manufacturing Technology, 2018, 97(5-8): 2419-2430.

[20] Xiao L, Anzai K, Niyama E, et al. Reducing "cold shut" defects in the " $\mathrm{H}$ " process aided by computer simulation. International Journal of Cast Metals Research, 1998, 11(2): 71-81.

[21] Vazquez V, Juarez-Hernandez A, Mascarenas A, et al. Cold shut formation analysis on a free lead yellow brass tap. Engineering Failure Analysis, 2010, 17(6): 1285-1289.

[22] Gao Z K, Chi Q X, Bu J L. Study on the crack failure mechanism of a turbine rear casing. Aeroengine, 2018, 44(6): 6-10. (In Chinese)

[23] Li S H. Crack analysis and optimization of casting spring pin on bottom plate of EMU. Foundry Technology, 2019, 40(10): 11271129. (In Chinese)

[24] Wu C Q, Liu Y K, Ma W H, et al. Fracture analysis of the screw hole on aluminum alloy casting. Heat Treatment of Metals, 2019 , 44(S1): 41-43. (In Chinese)

[25] Li N, Luo Z Q, Liu L, et al. Failure analysis of torsion axis. Heat Treatment of Metals, 2019, 44(S1): 255-257. (In Chinese)

[26] Hirt C W, Nichols B D. Volume of fluid (VOF) method for the dynamics of free boundaries. Journal of Computational Physics, 1981, 39(1): 201-225.

[27] Mirbagheri S M H, Dadashzadeh M, Serajzadeh S, et al. Modeling the effect of mould wall roughness on the melt flow simulation in casting process. Applied Mathematical Modelling, 2004, 28(11): 933-956. 